Technological University Dublin

DƯBLIN

ARROW@TU Dublin

2002-01-01

\title{
Holographically Recorded Photopolymer Diffractive Optical Element for Holographic and Electronic Speckle Pattern Interferometry
}

\author{
Sridhar Guntaka \\ Technological University Dublin \\ Vincent Toal \\ Technological University of Dublin, vincent.toal@tudublin.ie \\ Suzanne Martin \\ Technological University of Dublin, suzanne.martin@tudublin.ie
}

Follow this and additional works at: https://arrow.tudublin.ie/cieoart

Part of the Mechanical Engineering Commons

\section{Recommended Citation}

Guntaka, S., Toal, V. \& Martin, S. (2002) Holographically recorded photopolymer diffractive optical element for holographic and electronic speckle-pattern interferometry. Applied Optics, Vol. 41, no. 35, pp.

7475-7479. doi:10.1364/A0.41.007475

This Article is brought to you for free and open access by the Centre for Industrial and Engineering Optics at ARROW@TU Dublin. It has been accepted for inclusion in Articles by an authorized administrator of ARROW@TU Dublin. For more information, please contact arrow.admin@tudublin.ie, aisling.coyne@tudublin.ie, gerard.connolly@tudublin.ie.

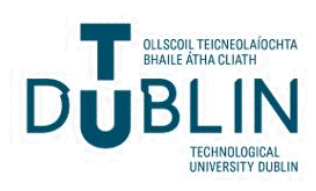




\title{
Holographically recorded photopolymer diffractive optical element for holographic and electronic speckle-pattern interferometry
}

\author{
Sridhar Reddy Guntaka, Vincent Toal, and Suzanne Martin
}

\begin{abstract}
A diffractive optical element is described that can be used to implement a very simple self-aligning electronic speckle-pattern interferometer and holographic interferometer that requires only a laser source and a camera in the optical setup. (C) 2002 Optical Society of America

OCIS codes: $\quad 070.0090,120.0120,090.2880,090.2890,120.6160$.
\end{abstract}

\section{Introduction}

Holographic interferometry and electronic specklepattern interferometry (ESPI) are closely related techniques with a wide range of applications in nondestructive evaluation, surface metrology, and defect detection. The advantage of holographic interferometry is the fringe quality that can be obtained. The disadvantage is that the hologram must be recorded and processed in situ, or the hologram must be relocated after processing, so that the reconstructed image and the illuminated object are exactly superimposed. In ESPI the hologram is replaced by a video camera and fringes can be obtained at TVframe rates, although the fringe quality is affected by speckle noise. In ESPI systems a reference beam and an object beam interfere on the faceplate of a video camera. In ESPI systems, which are sensitive to out-of-plane movement, the reference beam wavefront can be either smooth or speckled. In the former case it is necessary to ensure that the reference beam source is imaged at the center of the aperture stop of the imaging lens ${ }^{1}$ because of the very low spatial resolution of the CCD camera ( $~ 501$ $\left.\mathrm{mm}^{-1}\right)$. This requires a beam splitter between the imaging lens and the camera to combine the object and reference beams, which is wasteful of light. Al-

The authors are with the Centre for Industrial and Engineering Optics, School of Physics, Dublin Institute of Technology, Kevin Street, Dublin 8, Ireland. V. Toal's e-mail address is vincent.toal@dit.ie.

Received 7 June 2002; revised manuscript received 17 September 2002 .

0003-6935/02/357475-05\$15.00/0

(C) 2002 Optical Society of America ternatively, a mirror reflects the object wave into the camera. The mirror has a small aperture that allows the reference beam to be transmitted to the camera.

In the case of a speckled reference wave the light from the laser source is split into two beams in a Michelson interferometer. One beam illuminates the object and the other illuminates a fixed, optically rough surface. The light beams reflected by the object and the fixed surface are combined at the beam splitter and passed to the camera. This arrangement, although much easier to align, wastes at least half of the available light intensity.

ESPI is very similar to holographic interferometry, except that the CCD array takes the place of the high-resolution recording medium (hologram). The very limited resolution capability of the CCD $(\sim 50$ l. $\mathrm{mm}^{-1}$ ) is sufficient because, whether the reference wavefront is smooth or speckled, the optical setup used ensures that the object and reference beams are nearly coaxial as they enter the camera. The spatial frequency bandwidth of the resulting interference pattern is therefore centered at zero and the aperture is chosen so as to resolve the speckles.

In holographic recording the angle between the two recording beams is usually large enough to produce a much higher spatial-carrier frequency, in the recorded hologram. When live fringe holographic interferometry is performed, the low spatial frequency fringes observed in the hologram arise from the superposition of two nearly identical, superimposed wavefronts coming from the object and its holographic image.

In live fringe holographic interferometry it is essential that the reconstructed holographic image overlap the object precisely, before nondestructive 


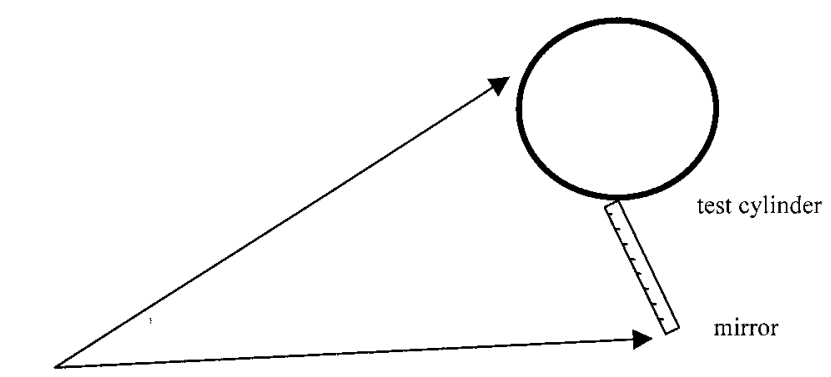

laser source

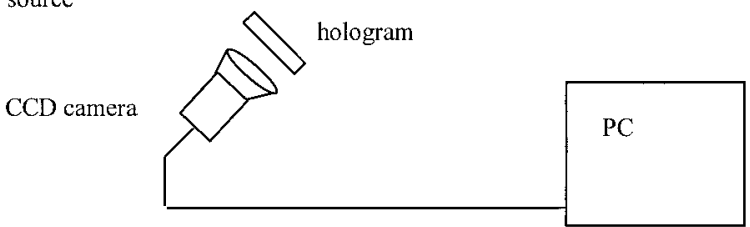

Fig. 1. Recording a transmission hologram of a test object for holographic interferometry and out-of-plane sensitive ESPI.

testing can begin. To achieve this, either the hologram must be relocated very precisely or any physical or chemical processing must be done in situ. Thermoplastic is an example of the former, while liquid gates can be used for silver halide chemical processing. Spurious fringes can arise due to emulsion shrinkage during processing, and although the highquality fringes obtained allow substantial movement before they become difficult to resolve, there is no possibility to start again as is possible with ESPI each time a new reference frame is captured.

In this paper we present a procedure for implementing live fringe holographic interferometry and out-of-plane sensitive ESPI in the same optical arrangement, in which ESPI can take over from holographic interferometry when fringes due to the latter become too fine to resolve.

\section{Transmission Holography and Electronic Speckle-Pattern Interferometry}

A transmission hologram was recorded by use of an air-cooled Argon ion laser of $100 \mathrm{~mW}$ output power operating at $514 \mathrm{~nm}$. The recording material is a self-processing photopolymer whose composition and holographic performance we have reported.2,3 It has also been used to make a diffractive optical element for use in an ESPI system. ${ }^{4}$ The object under test in this case was an unplasticised polyvinylchloride cylinder of inner radius $15.6 \mathrm{~cm}$, outer radius $18.6 \mathrm{~cm}$, and length $28.3 \mathrm{~cm}$, sealed at the ends and inflated by means of a hydraulic pump. Figure 1 shows the arrangement.

A pressure gauge and a $0^{\circ}, 45^{\circ}, 90^{\circ}$ rosette strain gauge were used to obtain mechanical test data. The rosette gauge provides values of the strain in three directions, allowing both maximum and minimum strains to be obtained. ${ }^{5}$ One hundred circular fringes appeared in the field of view as the pressure was raised from zero to 1 bar. From this a radial strain of 142 microstrain was calculated. The strain gauge result was also 142 microstrain leading to a

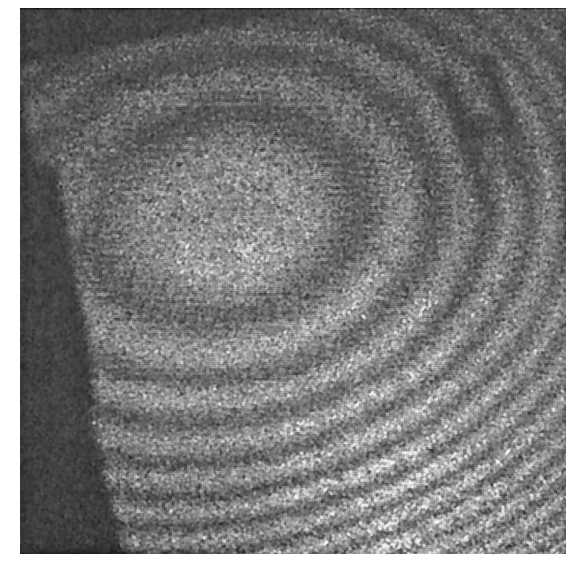

Fig. 2. Live holographic interferometric fringes of a radially expanding plastic cylinder using the arrangement of Fig. 1.

value of $2.78 \mathrm{GN} / \mathrm{m}$ for Young's modulus of elasticity, in reasonably good agreement with the manufacturer's data $\left(E\right.$ in the range $\left.2.8-3.2 \mathrm{GNm}^{-2}\right){ }^{6} \quad$ The experiment was repeated a number of times, with the same result and the fringe stability was also checked at a number of points in the pressure cycle. By this means one could be confident of the interferometric stability of the setup. A typical fringe pattern is shown in Fig. 2.

The hologram was now deliberately shifted so that no fringes could be generated by holographic interferometry. A CCD videocamera with a zoom lens was used to image both the object and the reconstructed holographic image. The double image was transferred to a frame store in a personal computer and, as the cylinder was inflated, the stored image was subtracted from incoming images. Circular ESPI fringes of low contrast were observed on the computer monitor (Fig. 3).

\section{Reflection Holography and Electronic Speckle-Pattern Interferometry}

The simplest implementation of a holographic interferometer involves the recording of a reflection or Denysiuk hologram of the object under test. For re-

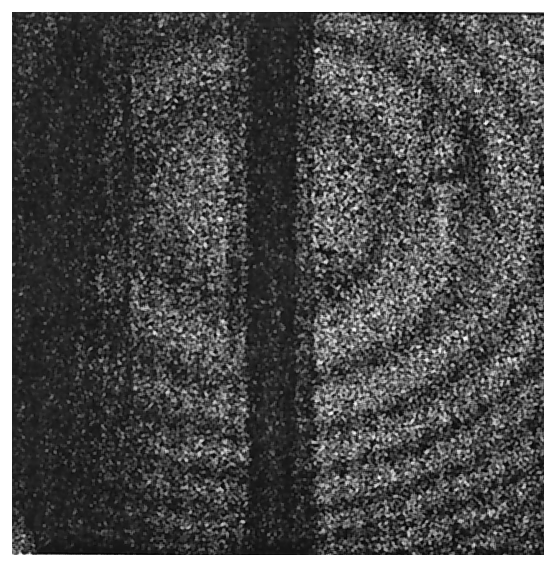

Fig. 3. Subtraction fringes in an out-of-plane ESPI system using the transmission hologram to generate the reference wavefront. 


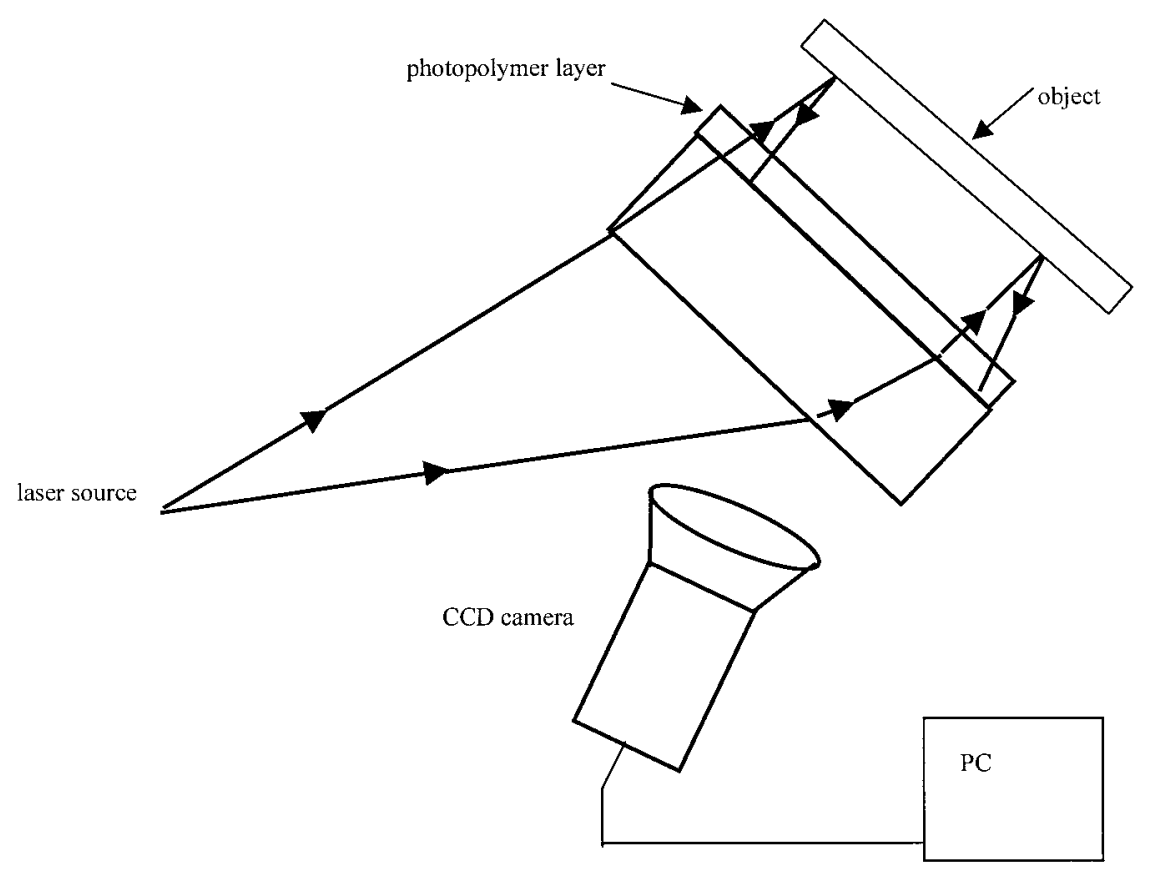

Fig. 4. Reflection hologram recording.

flection holography a He-Ne laser of $35 \mathrm{~mW}$ output power and HRT-BB panchromatic plate supported in a non-kinematic mount were first used. After processing, the hologram was replaced in the mount and slight adjustments were made until a clear bright holographic image was seen. As the cylinder was inflated, no fringes were seen, indicating that the object and holographically reconstructed image were not superimposed. When the holographically reconstructed image of the cylinder and the cylinder itself were imaged by the CCD camera, subtraction ESPI fringes were obtained as the cylinder was pressurized.

In another experiment a reflection hologram was recorded in a silver halide emulsion, of a flat, diffusely reflecting surface, and this hologram was used to generate the speckled reference beam in the outof-plane sensitive ESPI system as before. In the same way that a piece of ground glass can be used as a partial diffuser and can produce a suitable reference beam for out-of-plane sensitive ESPI, this hologram produces a speckled wavefront, with the object itself imaged through the hologram. In both trials the hologram was translated and rotated slightly, and the same results were obtained as before, which demonstrated a robustness not often obtained with out-of-plane sensitive ESPI and certainly not often obtained with holographic interferometry.

The photopolymer lacks the resolution required to record reflection holograms with high diffraction efficiency. However, it has qualities that would be very well suited to the present application. These include high angular selectivity and self-processing capability (for ease of use and alignment for holographic interferometry). In addition, the interferometer setup is very simple. With layers of thickness $125 \mu \mathrm{m}$, diffraction efficiency of over $90 \%$ is readily obtained. If such efficiencies could be obtained in reflection holography, then by rotating the hologram so that the reconstructing light is incident at an angle that differs from the Bragg angle, the brightness of the reconstructed image can be reduced to balance that of the illuminated object. This is important in out-of-plane ESPI because the object wave and reference waves have to be of equal intensity at the CCD array for optimum contrast in the subtractive fringe pattern.

Despite the low diffraction efficiency of the photopolymer at the spatial frequencies needed to record reflection holograms, it was thought worthwhile to try making reflection holograms for use in out-ofplane sensitive ESPI systems. Photosensitive layers of $120 \mu \mathrm{m}$ thickness were deposited on glass substrates, and the holograms were recorded very close to the object with the photosensitive layer facing the object. Figure 4 shows the arrangement. The object in this experiment was a vertically supported circular disk painted matt white, which could be rotated in its own plane by means of a remotecontrolled motor-driven encoder micrometer. Following the recording, the disk was rotated through a small angle and faint, live, holographic interferometric fringes were observed when we looked through the illuminated hologram. Illumination of the recorded hologram with the object removed also showed a weak but distinct reconstructed image of the disk.

To balance object-wave and reference-wave intensities for ESPI purposes, the disk was covered with a flat matt black plate and replaced in its original position. Figure 5 shows ESPI subtraction fringes due to rotation of the disk. 

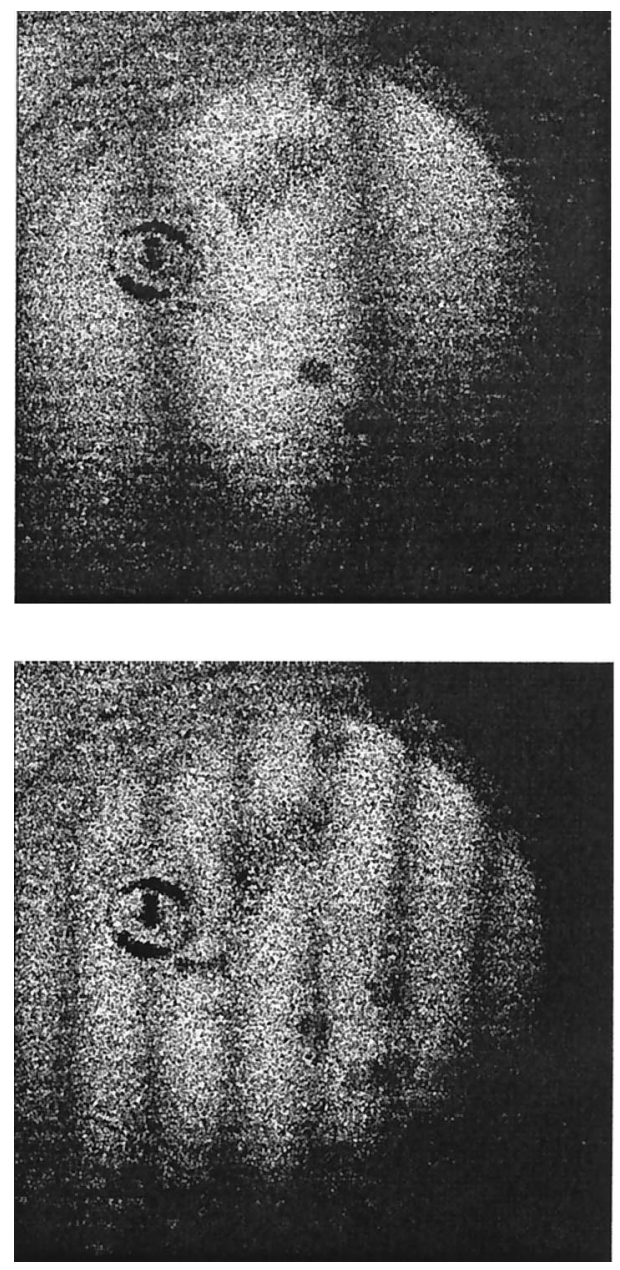

The main advantage of using a reflection hologram is simplicity in that the complete ESPI system now consists only of the laser, the hologram, and the video camera. A further advantage is that the hologram is a band-limited spectral filter and placing it immediately in front of the camera minimizes the effects of ambient light and maximizes the camera field of view.

\section{Discussion}

In these experiments we have recorded holographic diffractive optical elements that can be used to implement live fringe holographic interferometric nondestructive testing in the normal way but with the added feature that they serve as beam splitters and recombiners in extremely simple out-of-plane sensitive ESPI systems. The diffraction efficiency of the hologram can be altered by choosing exposure and processing in the case of silver halide or, in the case of the photopolymer, by simply rotating the hologram in position after recording it. The interferometer is a common-path one in the reflection hologram case and near common path in the transmission case, which increases immunity to temperature gradients. The reflection hologram can be recorded very close to the object for even greater interferometric stability or at some distance to provide for a large field of view.

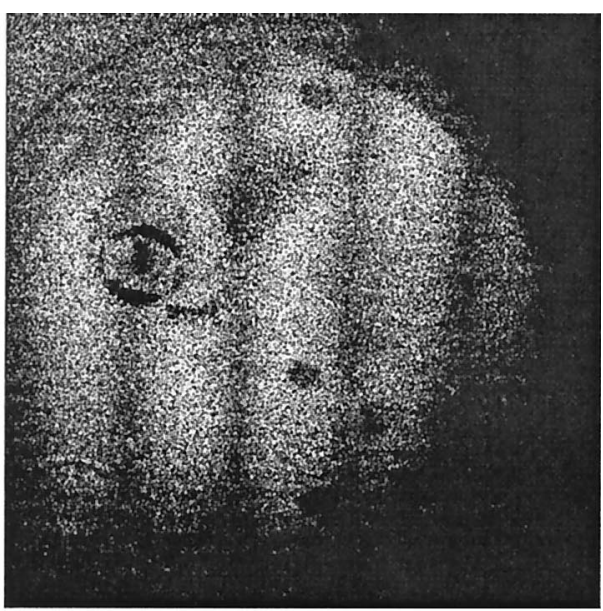

Fig. 5. Subtraction ESPI fringes obtained from photopolymer reflection hologram. The object is a flat circular disk rotated in its own plane.

As we have shown, it is not necessary that the hologram store an image of the actual object under test. A hologram of a flat surface can be prepared in advance and taken to the test site where ESPI measurements can be carried out. Alternatively, the photopolymer material can be used to record a hologram of the object at the test site under ambient lighting conditions rather than using momental processing of silver halide. ${ }^{7}$ It is also advantageous to use compact laser diodes in ESPI systems for reasons of portability and for this we anticipate recording the holograms in advance at visible wavelengths and replaying them on site using diode lasers with an output power of $50 \mathrm{~mW}$ and with a wavelength of 785 $\mathrm{nm}$. The spectral bandwidth of these holograms is sufficient to accommodate the shift in wavelength between recording and reconstruction. ${ }^{8}$ An additional feature is the implementation of digital speckle-pattern interferometry to carry out detailed fringe analysis. This can be implemented by altering the drive current in the laser diode to alter its wavelength and thus the optical path difference in the interferometer. Indeed, this is the only method by which phase shifting can be readily implemented in an ESPI system such as the present one, incorporating a reflection hologram.

An additional attractive feature of photopolymer- 
based holographic interferometry/ESPI is that as the number of fringes in holographic interferometry becomes too large to resolve, one can switch to ESPI and continue making measurements by refreshing the reference frame in the framestore.

Further work is aimed at improving the diffraction efficiency of the photopolymer at high spatial frequencies.

\section{Conclusion}

Simple combined holographic and electronic speckle pattern interferometers have been implemented by use of holographically recorded photopolymer diffractive optical elements. Such elements can be used as holograms for live fringe holographic interferometry and as beam splitters and combiners in electronic speckle pattern interferometry (ESPI). The ESPI systems are very simple and versatile, requiring a minimum of optical components. The holograms can be used at near infrared wavelength although recorded at visible wavelength.

\section{References and Note}

1. R. Jones, and C. Wykes, Holographic and Speckle Interferometry, 2nd ed. (Cambridge University, Cambridge, UK, 1985).

2. S. Martin, P. Leclere, Y. Renotte, V. Toal, and Y. Lion, "Characterisation of an acrylamide-based dry photopolymer holographic recording material," Opt. Eng. 33, 3942-3946 (1995).

3. S. Martin, C. A. Feely, and V. Toal, "Holographic characteristics of an acrylamide-based recording material," Appl. Opt. 36, 5757-5769 (1997).

4. M. P. Whelan, C. Forno, S. Martin, F. O Neill, and V. Toal, "Illumination systems using photopolymer gratings for speckle interferometry," in Interferometry '99 Applications, W. P. Jueptner and K. Patorski, eds., Proc. SPIE 3745, International conference on optical metrology, techniques and technologies, Warsaw, Poland, September $20^{\text {th }}$ to $23^{\text {rd }}, 1999$.

5. P. P. Benham, and F. V. Warnock, Mechanics of Solids and Structures, (Pitman, London, 1976).

6. Wavin Ireland Limited, Wavinmain Pressure System, 2000.

7. V. Petrov, and B. Lau, "Electronic speckle pattern interferometry with a holographically generated reference wave," Optical. Eng. 35, 2363-2370 (1996).

8. S. Reddy, V. Toal, and S. Martin, in Opto-Ireland 2002: Optics and Photonics Technologies and Applications, T. J. Glynn, ed., Proc. SPIE 4876, paper 173 (to be published). 